\title{
EL ROL DE LOS RECURSOS LABORALES EN EL DESARROLLO DEL ENGAGEMENT
}

\author{
M. Inmaculada Vicente de Vera García \\ Doctora en Prevención de Riesgos Laborales, investigadora \\ Universidad Pública de Navarra (UPNA) \\ ivicentedevera@yahoo.es \\ M. Inés Gabari Gambarte \\ Doctora en Psicología \\ Profesora Titular de Universidad \\ del Área Didáctica y Organización Escolar \\ Universidad Pública de Navarra (UPNA) \\ igabari@unavarra.es
}

Fecha de Recepción: 2 Septiembre 2019

Fecha de Admisión: 25 Septiembre 2019

\section{RESUMEN}

Los factores psicosociales en el trabajo son determinadas condiciones directamente relacionadas con el contenido del puesto, las exigencias de la realización de la tarea e incluso con el entorno, que tienen capacidad de afectar el desarrollo del trabajo de los profesionales. Cuando estos factores tienen consecuencias perjudiciales para la seguridad, salud (física, psíquica o social) y bienestar, hablamos de factores de riesgo psicosocial o fuentes de estrés laboral. El estudio del burnout como factor de riesgo psicosocial es una necesidad orientada a mejorar la salud y la calidad de vida de los trabajadores en las organizaciones. No obstante, las últimas tendencias en el estudio del burnout han dado un giro hacia el estudio del engagement como aspecto positivo y óptimo del desarrollo profesional y, en este sentido, entre las principales causas del engagement destacan los recursos laborales. En el presente trabajo se realiza un recorrido sobre la evolución de la investigación sobre burnout hacia su opuesto, el engagement, y se describen los principales recursos laborales que contribuyen a su desarrollo y al bienestar psicosocial de los empleados en las organizaciones actuales, según recoge la literatura.

Palabras clave: burnout; engagement; psicología positiva; recursos laborales.

\section{ABSTRACT}

The role of labor resources in the development of Engagement. Psychosocial factors at work are certain conditions directly related to the content of the position, the requirements of the realization of the task and even with the environment, which have the capacity to affect the development of the work of the Professional. When these factors have detrimental consequences for your safety, health (physical, psychic or social) and wellbeing, we talk about psychosocial risk factors or sources of work stress. The study of burnout as a psychosocial risk factor is a need aimed at improving the health and quality of life of workers in organizations. However, the latest trends in the study of burnout have taken a turn towards the study of engagement as a positive and optimal aspect of professional development and, in this sense, among the main causes of engagement work resources. In 


\section{EL ROL DE LOS RECURSOS LABORALES EN EL DESARROLLO DEL ENGAGEMENT}

the present work, a tour of the evolution of burnout research towards its opposite, engagement, and the main labor resources that contribute to its development and the psychosocial well-being of employees in current organizations are described, according to the literature.

Keywords: burnout; engagement; positive psychology; labor resources.

\section{INTRODUCCIÓN}

Los profesionales se ven expuestos continuamente a diferentes estresores en las instituciones. Dependiendo de la percepción de cada sujeto, éstos pueden generar conductas de adaptación para superar dichas circunstancias amenazantes y así sobrellevar el trabajo, 0 bien mostrar conductas desadaptativas que generan gran desgaste físico y emocional (Medrano, 2017; Vicente de Vera y Gabari, 2019). La respuesta de cada individuo ante estas situaciones es acorde al ambiente que existe en la institución, ya que éste impacta en el tipo de estrés que experimenta su personal y, por ende, repercute en su productividad. favorece la existencia de personal productivo, motivado, leal y comprometido con la organización (Montoya, Hincapié y Carmona, 2016; Alarcón, 2018). Por el contrario, cuando el ambiente laboral es negativo, poco motivador o con una carga de trabajo excesiva para las capacidades de los empleados y empleadas, estos comienzan a manifestar la presencia del estrés que, cuando se produce de manera crónica, puede dar lugar al desarrollo del burnout (Aldás, 2017; Peña, Raso y Ferrero, 2018).

El burnout, también llamado síndrome de estar quemado, se caracteriza por un progresivo deterioro de la comunicación y de las relaciones interpersonales en el trabajo. El sujeto que lo padece alberga sentimientos que oscilan desde la indiferencia a la desesperación, disminuye la productividad y la calidad del servicio prestado, con frecuencia se da el absentismo y la desmotivación y aumentan los deseos de dejar ese empleo por otra ocupación, aspectos que generan en la persona una creciente desmoralización (Verduzco y Hernández, 2018).

Sin embargo, las organizaciones de hoy en día precisan todo lo contrario. Necesitan que sus trabajadores y trabajadoras estén motivados, que sean proactivos, que colaboren con los demás y que estén implicados en el trabajo y en la institución para la cual trabajan (Gómez, Meneses y Palacio, 2017). Las empresas que pretenden alcanzar estabilidad y crecimiento deben contemplar que para sus empleados y empleadas hay factores esenciales como: 1) el desarrollo que se les ofrece, 2) la calidad de vida que se les genera, 3) la motivación implícita, 4) los incentivos para aumentar el deseo de permanecer en la organización, 5) la valoración y énfasis de su labor y 6) el reconocimiento a la importancia de sus funciones. A través de estos factores se favorecen actitudes de compromiso y entusiasmo con el trabajo realizado (Acosta, Torrente, Llorens y Salanova, 2015).

Es fundamental que las instituciones se esfuercen, no sólo por cumplir sus objetivos, sino los de sus trabajadores y trabajadoras (Giraudier, 2018). Es necesario dejar de considerar a los y las empleados como instrumentos para conseguir los fines empresariales y aproximarse a la concepción de la Psicología Organizacional Positiva que entiende la salud del trabajador como meta en sí misma y como objetivo legítimo a incluir en las políticas organizacionales (Acosta, Salanova y Llorens, 2013; Acosta, Oramas, del Castillo y Vergara, 2017).

La Psicología Organizacional Positiva pone énfasis en los recursos laborales y en su potencial motivador, uno de estos recursos es, precisamente, estar comprometido con la tarea (González, Torres y Carreres, 2017). Así, desde el estudio del estado mental negativo relacionado con el trabajo (burnout), la investigación ha orientado su interés por el estudio de un estado psicológico positivo que incluye las fortalezas personales y presta mayor atención a las experiencias positivas de los y las profesionales y a las condiciones que favorecen su bienestar en la empresa, temáticas con frecuencia olvidadas en el ámbito laboral (Cifre, Castellano, Spontón, Medrano y Maffeic, 2017). Este estado psicológico positivo relacionado con el trabajo es: el engagement.

Dado que el engagement incluye características tales como el compromiso, la motivación, el entusiasmo, el esfuerzo y todos aquellos aspectos que propician vínculos positivos entre el empleado y la organización, a continuación, nos centramos en su definición. 


\section{CONCEPTO DE ENGAGEMENT}

El engagement, es considerado como el concepto teóricamente opuesto al burnout o síndrome de estar quemado (Maslach y Leiter, 1997). El burnout, es un síndrome complejo compuesto por tres dimensiones básicas: agotamiento emocional, despersonalización o cinismo y falta de eficacia profesional. En términos generales, el engagement o vinculación psicológica en el trabajo se refiere a un estado afectivo-cognitivo persistente que no está focalizado en un objeto, evento o situación particular (Schaufeli, Salanova, Gonzalez-Romá y Bakker, 2002a:72). La investigación empírica realizada sobre el engagement ha identificado tres dimensiones: el vigor, la dedicación y la absorción (Lugo, 2016).

El vigor se caracteriza por altos niveles de energía, resistencia y activación mental mientras se trabaja, así como por la predisposición de invertir esfuerzo y la persistencia, incluso cuando aparecen dificultades. La dimensión dedicación denota alta implicación laboral y alto nivel de significado atribuido al trabajo. Finalmente, la absorción, ocurre cuando se está totalmente concentrado en la tarea, de manera que se tiene dificultad a la hora de desconectar de lo que se está haciendo debido a las fuertes dosis de disfrute y concentración experimentadas. Vigor y dedicación son considerados los opuestos de las dimensiones del burnout. agotamiento y cinismo, respectivamente (Martos et al., 2018). Con respecto a la absorción, algunos autores afirman que puede considerarse más una consecuencia del engagement que una de sus dimensiones (Durango, 2018).

En definitiva, cuando se analizan los componentes del engagement se advierte que responde a un constructo claramente motivacional ya que posee componentes de activación, energía, esfuerzo y persistencia y está dirigido a la consecución de objetivos (de la Fuente, Sevillano, Sander y Putwain, 2016). Por ello, se considera un indicador de la motivación intrínseca por el trabajo (Samanez, Stephani y Placencia, 2017). De modo que para los profesionales comprometidos su trabajo es algo positivo porque disfrutan con y de lo que hacen. En cambio, los profesionales quemados se sienten agotados y fatigados, tienen actitudes cínicas y escépticas sobre su trabajo y se sienten mal en ellos (Brito, 2018).

En suma, el engagement es un estado psicológico caracterizado por altos niveles de energía y una fuerte implicación con el trabajo; mientras que el burnout, se caracteriza por el opuesto: bajos niveles de energía combinados con una falta de identificación con el propio trabajo (Bakker, 2011; García, Escorcia y Suarez, 2017; Vicente de Vera y Gabari, 2019).

La siguiente Tabla muestra una comparativa de las dimensiones que componen ambos constructos. 
Tabla 1. Dimensiones del burnout vs dimensiones del engagement.

\title{
DIMENSIONES DEL BURNOUT DIMENSIONES DEL ENGAGEMENT
}

Agotamiento emocional: se caracteriza Vigor: se refiere a la energía como por cansancio y fatiga física, psíquica o opuesto al agotamiento. Existe una combinación de ambos. Es la sensación de activación mental en el trabajo, la voluntad no poder dar más de sí mismo a los demás y predisposición de invertir esfuerzos

Despersonalización: se caracteriza por el Dedicación: consiste en involucrarse, desarrollo de sentimientos, actitudes y entusiasmarse, estar orgulloso e inspirado respuestas negativas, distantes y frías en el trabajo. Se caracteriza por un hacia otras personas. Existe irritabilidad y sentimiento de importancia y desafío una pérdida de motivación

\begin{abstract}
Bajo logro o realización profesional y/o Absorción: se caracteriza por un estado de personal: surge cuando se verifica que las concentración, sensación de que el tiempo demandas que se le hacen al individuo pasa volando, en el que el sujeto tiene exceden su capacidad para atenderlas de dificultades para desligarse del trabajo
\end{abstract}

forma competente. Hay un bajo rendimiento laboral, incapacidad para soportar la presión y una baja autoestima. Se experimentan sentimientos de fracaso personal

La literatura científica en el tema subraya que el engagement tiene repercusiones positivas tanto para el individuo como para la organización (Fernández, 2017). A nivel individual proporciona fuerte identificación con el trabajo, buena salud mental y bajo riesgo de quemarse, genera buen desempeño, aumenta la motivación intrínseca y la adquisición de recursos laborales y personales como la autoeficacia.

Desde este punto de vista organizacional, el engagement está positivamente relacionado con el compromiso organizacional (Castrejón, 2016). También se relaciona positivamente con la conducta extra-rol, la iniciativa personal, el rendimiento y la calidad del servicio (Extremera, Bravo y Durán, 2016). En cambio, la relación es negativa con el absentismo, la rotación y la tendencia a abandonar la organización (Polo, Fernández, Bargsted, Fama y Rojas, 2017). En este sentido, Pulido y Herrera (2018) constatan que bajos niveles de compromiso en la ocupación están asociados con altos niveles de burnout.

La integración de las personas en las organizaciones es primordial, pues de este acoplamiento depende que los intereses, los objetivos y las necesidades de cada cual Ileguen a un nivel armónico que logre que la relación empleado-empresa perdure en el tiempo (Espinosa, Icaza, Sadi, y Adell, 2017). Según estos autores, el compromiso organizacional permite estabilidad laboral ya que impacta en prestaciones, jubilación, beneficios sociales y garantiza la cobertura de necesidades tanto materiales como psicológicas (reconocimiento, desarrollo de habilidades, socialización, estatus).

A continuación, se realiza una revisión de aquellos recursos laborales que los estudios constatan como facilitadores del desarrollo del engagementy contribuyen a la prevención del llamado síndrome de burnout.

\section{RECURSOS LABORALES QUE FAVORECEN EL ENGAGEMENT}

El origen del burnout se emplaza con elevadas demandas laborales, las cuales provocan deterioro en la salud del trabajador y la trabajadora. Por contra, el engagement, se relaciona con la disponibilidad de recursos laborales, lo que favorece mayor compromiso con la organización (Ospina y Meneghel, 2017). 
En general, en casi cualquier tipo de trabajo podemos apreciar la existencia de dos elementos: demandas y recursos laborales. Las demandas son características del trabajo que requieren esfuerzo del empleado/empleada para realizarlas. Los recursos laborales tienen un papel esencial para hacer frente a dichas demandas, ya que actúan como motivadores estimulando el engagement y la iniciativa de los y las trabajadores y, con el tiempo, el engagementy la iniciativa personal dan paso a más recursos (Lozano y Reyes, 2017). De modo que, cuantos más recursos laborales están disponibles, mayor es la probabilidad de tener profesionales vinculados psicológicamente con la organización (Suarez y Díaz, 2015).

Los recursos laborales se refieren a aspectos a dos niveles: a) a nivel de la institución (ej., paga, seguridad en el puesto, oportunidades para el desarrollo de carrera), b) a nivel interpersonal y relaciones sociales (ej., apoyo de la supervisión, clima psicosocial del equipo), recursos relacionados con la organización del trabajo (ej., claridad de rol, participación en la toma de decisiones y recursos relacionados con la tarea (ej., variedad, autonomía, feedback). Todos ellos, facilitan la consecución de objetivos y reducen los costes físicos y psicológicos asociados a las demandas estimulando el desarrollo y el crecimiento personal (Pujol y Arraigada, 2017). En sentido contrario, la falta o inadecuación de recursos laborales incrementa las demandas y, por ende, contribuye indirectamente a la génesis de estresores.

La oportunidad que se ofrece para que el sujeto utilice y desarrolle sus propias habilidades es un recurso laboral primordial. Las habilidades se entienden como la capacidad cognitiva necesaria para desempeñar las funciones del puesto de trabajo. Los estudios constatan que las empresas que favorecen el uso de las habilidades obtienen trabajadores y trabajadoras más satisfechos, con niveles de autoestima más altos y con mayores indicadores de bienestar psicosocial (González, González y San José, 2017). Asimismo, la presencia de demandas laborales realistas genera puestos activos y motivadores que fomentan el desarrollo personal. Pero si existen excesivas demandas en relación con las competencias, conocimientos y habilidades de la persona, aparecen problemas de estrés (Rodríguez, Sola y Fernández, 2017). De igual modo, cuando el trabajo requiere pocas tareas 0 éstas son muy simples, rutinarias y aburridas en relación con las habilidades y destrezas del sujeto, también ello puede perjudicar su salud (Vallejo, 2017).

Además, es fundamental que el/la profesional conozca cuál es su rol en la institución para la que trabaja. Ha de recibir información clara respecto a lo que se espera de él/ella, sobre la conducta requerida, sobre el resultado de la realización de sus tareas y sobre su futuro en la organización (Mone y London, 2014; Peña y Cantero, 2017).

La autonomía en el puesto es clave en la mayoría de los modelos de estrés (Burgos, Paris, Salcedo y Arriagada, 2018). Dicho recurso tiene dos aspectos básicos: a) control de tiempos y b) control de métodos. El control de tiempos hace alusión al grado en que el sujeto puede decidir cuándo llevar a cabo una determinada tarea. El control de métodos se refiere a la capacidad que tiene el y la profesional para decidir cómo va a realizar el trabajo.

Adicionalmente, en la realización del trabajo ha de existir variedad de tareas (Hernández y Viera, 2018). Se distinguen dos tipos: variedad intrínseca y variedad extrínseca. La variedad intrínseca es el grado en que una tarea requiere diversas actividades para llevarla a cabo e implica el uso de diferentes habilidades. La variedad extrínseca tiene que ver con los aspectos relacionados con el entorno laboral, como son: la música ambiental, cambios en la iluminación o la oportunidad de mirar al exterior.

En este marco, la percepción de apoyo social es crucial para el individuo (Orgambídez y Borrego, 2017). Saber que se puede contar con el apoyo de los compañeros o de la supervisión del servicio se considera un recurso organizacional imprescindible. Este puede ser: a) de tipo emocional, a través de sentimientos de empatía, estima y confianza o b) de tipo instrumental, por ejemplo ayudando a un compañero/compañera cuando le surge un problema (Murillo, Garrido y Hernández, 2016). Si tenemos en cuenta que gran parte de nuestro tiempo lo pasamos en el trabajo, las relaciones interpersonales con los compañeros/compañeras y clientes/usuarios cobran especial relevancia para nuestra identidad como personas y para nuestro equilibrio físico y mental (Carlotto y Câmara, 2017). 


\section{EL ROL DE LOS RECURSOS LABORALES EN EL DESARROLLO DEL ENGAGEMENT}

Por último, es preciso tener información sobre el trabajo realizado. El feedback hace referencia al grado en que la realización de la actividad laboral proporciona a la persona información clara y directa sobre la eficacia de su desempeño. Este puede ser proporcionado por la propia supervisión o por los compañeros/compañeras de trabajo. El feedback, es responsable de la aparición de consecuencias positivas, tales como el incremento en las creencias de eficacia, el bienestar psicológico, la satisfacción y la productividad (Winstone, Nash, Parker y Rowntree, 2017). Así, cuando el sujeto percibe una falta de reciprocidad en el intercambio social decimos que se ha violado el contrato psicológico. Por el contrario, cuando lo que existe es reciprocidad, el contrato psicológico se mantiene vigente (Orozco, 2016).

Si el sujeto empleado está vinculado emocional y conductualmente con la organización, aumenta la productividad y los/las clientes sienten satisfacción con la atención que se les brinda. El vínculo emocional que se produce en los individuos refleja variables como: cumplimiento de logros y metas, trabajo en equipo, buen control del tiempo, buenas relaciones humanas y una labor eficaz enfocada a grandes resultados. Por este motivo, el personal encargado de la supervisión, así como los mandos intermedios, juegan un papel relevante como ejecutores de los diversos contratos psicológicos (Lisbona, Palaci, Salanova, Frese, 2018).

En síntesis, el hecho de mantener vigente el contrato psicológico entre empleado/empleada y organización emerge como una técnica útil para la optimización del engagement y como sinergia para evitar el burnout (Hurtado y Pereira, 2015). De hecho, estudios realizados con diversas muestras de profesionales demuestran la influencia que tiene la presencia de conflictos interpersonales y la falta de reciprocidad en los intercambios sociales del ambiente laboral y en la génesis y desarrollo de la sintomatología del burnout (Vázquez, Casal y Pérez, 2018).

\section{CONCLUSIONES Y RECOMENDACIONES}

En este artículo hemos pretendido ofrecer una panorámica sobre el paradigma de la psicología positiva aplicado al ámbito de las organizaciones modernas. Hemos señalado cuáles son las mejores organizaciones para trabajar, aquellas que cuidan el contexto de trabajo y realizan las mejores prácticas.

Concluimos que el engagementes un estado psicológico positivo que perdura en el tiempo, incluye aspectos tales como energía y dedicación al trabajo -aspectos positivos emocionales de los y las profesionales- y una condición de salud. Si la organización brinda a sus empleados y empleadas bienestar, no sólo a nivel físico, sino también a nivel psicológico, estos y estas se sienten comprometidos mostrando agrado y pasión por su trabajo. Por tanto, cuando el y la profesional están comprometidos con su trabajo y con su organización dedicando sus esfuerzos e ilusiones a lograr su realización laboral, existen menos posibilidades de que aparezca el burnout. El sujeto comprometido siente un fuerte deseo de permanecer en la institución, reconoce que sus ideas son importantes para el desarrollo de la empresa y ello facilita la retención de talentos. En definitiva, parece que todo apunta a que los y las empleados deciden comprometerse con la institución en la que trabajan si se encuentran contentos con la misma.

Como ha quedado descrito, las organizaciones positivas lo son en la medida en que los puestos cuentan con recursos laborales específicos. Concluimos, que los recursos laborales mantienen una relación recíproca con el engagement. La disponibilidad de dichos recursos estimula el desarrollo personal, la motivación y el bienestar psicológico de los y las profesionales en forma de engagement. Como consecuencia, se obtienen resultados positivos en el trabajo, compromiso organizacional, buen desempeño, así como reducción de la intención de abandono. En suma, el resultado que se establece entre los recursos laborales y el engagement es la ganancia.

Consideramos que los programas de intervenciones positivas deben formar parte integrante de la política y la cultura de las instituciones que valoran, propician y potencian la salud y el bienestar de sus trabajadores y trabajadoras. Es preciso pasar de la concepción del lugar de trabajo como un espacio para el intercambio comercial, mercantil o productivo a entenderlo como un espacio de vida, en el cual las personas contribuyen con sus esfuerzos, energía y competencias para que la organización alcance sus objetivos que, al final, son los objetivos com- 
partidos y considerar que el personal trabajador, así como los cargos de dirección, deben colaborar para convertirlo en un entorno saludable donde se propicie la salud en toda la extensión de la palabra.

\section{REFERENCIAS BIBLIOGRÁFICAS}

Acosta, H., Salanova, M. \& Llorens, S. (2013). Building organizational trust: A study in small and medium-sized enterprises. In J. Neves \& S. P. Gonçalves (Eds.), Occupational health psychology: From Burnout to wellbeing (pp. 357-378). Rosemead, CA: Scientific \& Academic Publishing.

Acosta, H., Torrente, P., Llorens, S. \& Salanova, M. (2015). La confianza es pasión: La relación entre confianza organizacional y el engagement de los equipos. Revista Latinoamericana de Psicología Positiva, 2(1), 7-21.

Alarcón, M. E. B. (2018). Estrés y Burnout Enfermedades En La Vida Actual. Palibrio.

Aldás, A. C. (2017). Estrategias de afrontamiento y síndrome de burnout en personal de salud (thesis, Universidad Técnica de Ambato-Facultad de Ciencias de la Salud-Carrera de Psicología Clínica). Recuperado de: http://repo.uta.edu.ec/bitstream/123456789/26500/2/Ana\%20Carolina\%20Ald\%C3\%A1s\%20Villac\%C3\% ADs\%20pdf.pdf

Bakker, A. B. (2011). An evidence-based model of work engagement. Current Directions in Psychological science, 20(4), 265-269. doi.org/10.1177/0963721411414534

Brito, J. (2018). Calidad educativa en las instituciones de educación superior: evaluación del síndrome de burnout en los profesores. RIDE. Revista Iberoamericana para la Investigación y el Desarrollo Educativo, 8(16), 516-534

Burgos, A. L. V., Paris, A. P. D., Salcedo, A. G. S. \& Arriagada, A. A. (2018). Psychological well-being and burnout in primary health care professionals in the region of Los Lagos, Chile. Acta Universitaria, 28(3), 56-64.

Carlotto, M. S. \& Câmara, S. G. (2017). Psychosocial Risks Associated with Burnout Syndrome Among University Professors. Avances en Psicología Latinoamericana, 35(3), 447-457. doi.org/10.12804/revistas.urosario.ed u.co/apl/a.4036

Castrejón, M. C. (2016). Relación entre los niveles de engagement y compromiso organizacional en personal de una institución de educación superior de la ciudad de Cajamarca 2013. Revista Tendencias en Psicología, $1(2), 45-52$.

Cifre, E., Castellano, E., Spontón, C., Medrano, L. A. \& Maffeic, L. (2017). Emociones positivas y negativas en la predicción del burnout y engagement en el trabajo. Revista Peruana de Psicología y Trabajo Social, 2(1), 7588.

de la Fuente, J., Sevillano, L. Z., Sander, P. \& Putwain, D. (2016). El papel de la enseñanza reguladora en la inducción de las emociones de engagement-bournot del alumnado universitario. International Journal of Developmental and Educational Psychology (Revista INFAD de Psicología), 4(1), 149-154. doi.org/10.17060/ijodaep.2014.n1.v4.598

Durango, B. (2018). Bienestar psicológico como elemento principal para el engagement. CICAG, 14(1), 131-142.

Espinosa, N. W., Icaza, M. I. C., Sadi, G. \& Adell, I. A. (2017). La relación entre la satisfacción con la comunicación interna y el compromiso organizacional: el caso de una universidad ecuatoriana. Austral Comunicación, 6(1), 133-160. doi.org/10.26422/aucom.2017.0601.wil

Felix, R. 0., García, C. \& Mercado, S. M. (2018). Stress in the workplace Generic review from the theory. CULCyT, 64(1), 31-42.

Fernández, M. P. (2017). Relación entre Desgaste ocupacional y manifestaciones psicosomáticas en una muestra de docentes colombianos de la ciudad de Santa Marta. Archivos de Medicina (Manizales), 17(1), 78-90.

García, A. A., Escorcia Bonivento, C. V. \& Perez, B. S. (2017). Burnout Syndrome and Self-Efficacy Beliefs in Professors. Journal of Educational Psychology-Propositos y Representaciones, 5(2), 65-126. doi.org/10.20511/pyr2017.v5n2.170 


\section{EL ROL DE LOS RECURSOS LABORALES EN EL DESARROLLO DEL ENGAGEMENT}

Giraudier, M. (2018). Cómo gestionar el clima laboral. Manuel Giraudier.

Gómez, G. E., Meneses, A. C. \& Palacio, M. C. (2017). La satisfacción laboral y el capital psicológico: factores que influyen en el síndrome de burnout. Ansiedad y Estrés, 23(2-3), 71-75. doi.org/10.1016/j.anyes.20 17.09.002

González, R. P., González, M. J. A. \& San José, C. C. (2017). Afectividad positiva y negativa en el futuro docente: relaciones con su rendimiento académico, salud mental y satisfacción con la vida. Contextos Educativos. Revista de Educación, 20(1), 11-26. doi.org/10.18172/con.2996

González, T. M. P., Torres, L. H. \& Carreres, A. L. (2017). Fortalezas y virtudes personales del profesorado y su relación con la eficacia docente. International Journal of Developmental and Educational Psychology (Revista INFAD de Psicología), 7(1), 141-150. doi.org/10.17060/ijodaep.2014.n1.v7.785

Hernández, L. H. \& Viera, A. 0. (2018). Factores psicosociales laborales relacionados con el work engagement desde el modelo de Demanda-Recursos laborales en trabajadores cubanos. Revista Cubana de Salud y Trabajo, 19(2), 19-23.

Hurtado, D. \& Pereira, F. (2015). El síndrome de desgaste profesional (burnout Syndrome): manifestación de la ruptura de reciprocidad laboral. Revista Salud Bosque, 2(2), 29-38. doi.org/10.18270/rsb.v2i2.61

Lisbona, A., Palaci, F., Salanova, M. \& Frese, M. (2018). The effects of work engagement and self-efficacy on personal initiative and performance. Psicothema, 30(1), 89-96.

Lozano, C. R. \& Reyes, M. (2017). Docentes universitarios: Una mirada desde la Autoeficacia general y engagement laboral. Revista Digital de Investigación en Docencia Universitaria, 11(1), 134-148. doi.org/10.19083/ridu.11.503

Lugo, M. E. M. (2016). La vinculación psicológica en el trabajo (engagement), el síndrome de quemarse por el trabajo y el clima organizacional en un grupo de empleados en Puerto Rico. Revista interamericana de psicología ocupacional, 28(1), 7-15.

Martos, Á., del Carmen Pérez, M., del Mar Molero, M., Gázquez, J. J., del Mar Simón, M. \& Barragán, A. B. (2018). Burnout y engagement en estudiantes de Ciencias de la Salud. European Journal of Investigation in Health, Psychology and Education, 8(1), 23-36. doi.org/10.30552/ejihpe.v8i1.223

Maslach, C. \& Leiter, M. P. (1997). The truth about burnout: How organizations cause personal stress and what to do about it. San Francisco: Jossey-Bass.

Medrano, F. M. (2017). Estilos de afrontamiento del estrés que aplica el profesional de enfermería en el servicio de emergencia del Hospital Nacional Daniel Alcides Carrión mayo-2016. Recuperado de http://repositorio.upsjb.edu.pe/handle/upsjb/1368

Mone, E. M. \& London, M. (2014). Employee engagement through effective performance management: A practical guide for managers. New York: Routledge/Taulor\& Francis.

Suárez, N. \& Díaz, L. B. (2015). Estrés académico, deserción y estrategias de retención de estudiantes en la educación superior. Revista de salud pública, 17(1), 300-313.

Murillo, F. J., Garrido, C. M. \& Hernández, R. (2016). Decalogue for effective teaching. REICE. Ibero-American Journal on Quality, Efficacy and Change in Education, 9(1), 6-27.

Oramas, A., del Castillo, N. P. \& Vergara, A. (2017). Dos modos de vinculación con el trabajo: compromiso (work engagement) y adicción. Relación con bienestar físico y psicológico. Revista Cubana de Salud y Trabajo, 18(3), 23-34.

Orgambídez, A. \& Borrego, Y. (2017). Apoyo social y engagement como antecedentes de la satisfacción laboral en personal de enfermería. Enfermería Global, 16(48), 208-225. doi.org/10.6018/eglobal.16.4.260771

Orozco, C. A. C. (2016). Síndrome de burnout en docentes universitarios. INNOVA Research Journal, 1(9), 7795.

Ospina, J. L. \& Meneghel, I. (2017). Clima Organizacional como antecedente del Engagement en una muestra de empresas colombianas. Revista Interamericana de Psicología Ocupacional, 35(1), 21-34. doi.org/10.21772/r ipo.v35n1a02 
Peña, J. E., Raso, P. C. \& Ferrero, B. S. (2018). El síndrome de burnout en los docentes. Ediciones Pirámide.

Peña, S. B. \& Cantero, V. F. (2017). Prejuicio étnico y desgaste docente: un desafío en contextos de pobreza. Revista Electrónica Diálogos Educativos, 10(20), 14-39.

Polo, J. D., Fernández, M., Bargsted, M., Fama, L. F. \& Rojas, M. (2017). Relación entre el compromiso organizacional y la satisfacción con la vida: La mediación del engagement. Universia Business Review, 54(1), 110145.

Pujol, L. \& Arraigada, M. (2017). Propiedades psicométricas del Cuestionario de Riesgos Psicosociales CopsoqIstas 21 y aplicación en docentes universitarios argentinos. Cuadernos de Administración, 30(55), 97-125.

Pulido, F. \& Herrera, F. (2018). Predictors of Happiness and Emotional Intelligence in Secondary Education. Revista Colombiana de Psicología, 27(1), 71-84. doi.org/10.15446/rcp.v27n1.62705

Rodríguez, A. M., Sola, T. \& Fernández, M. (2017). Impacto del Burnout en el desarrollo profesional del profesorado universitario. Una revisión de la investigación. Revista Electrónica Interuniversitaria de Formación del Profesorado, 20(3), 161-178. doi.org/10.6018/reifop.20.3.275121

Samanez, M., Stephani, H. \& Placencia, M. D. (2017). Motivación y satisfacción laboral del personal de una organización de salud del sector privado. Horizonte Médico, 17(4), 42-52. doi.org/10.24265/horizmed.20 17.v17n4.08

Schaufeli, W. B., Salanova, M., González, V. \& Bakker, A. B. (2002a). The measurement of engagement and burnout. A two sample confirmatory factor analytic approach. Journal of Happiness studies, 3(1), 71-92. doi.org/10.1023/A:1015630930326

Vallejo, M. (2017). Una aproximación al síndrome de burnout y las características laborales de emigrantes españoles en países europeos. Revista de Psicología del Trabajo y de las Organizaciones, 33(2), 137-145. doi.org/10.1016/j.rpto.2017.02.001

Vázquez, G. V., Casal, C. C. \& Pérez, D. Á. (2018). Liderazgo Transformador, Engagement Y Creatividad En El Contexto De Pymes Intensivas En Conocimient. Revista Galega de Economía, 27(1), 65-80.

Vicente de Vera, M. I. \& Gabari, M. I. (2019). "Potential of teacher resilience before educational changes". International Journal of Development Research, 09(03), 26712-26718.

Vicente de Vera, M. I. \& Gabari, M. I. (2019). Liderazgo Pedagógico en Educación Secundaria: Aportaciones desde la Evaluación de Burnout-Resiliencia en Docentes. International Journal of Educational Leadership and Management, 7(1), 104-134. doi.org/10.17583/ijelm.2018.3519

Vicente de Vera, M. I. \& Gabari Gambarte, M. I. (2019). Burnout y Factores de Resiliencia en Docentes de Educación Secundaria, International Journal of Sociology of Education, 8(2), 127-152. doi.org/ 10.17583/rise.2019.3987

Winstone, N. E., Nash, R. A., Parker, M. \& Rowntree, J. (2017). Supporting learners' agentic engagement with feedback: A systematic review and a taxonomy of recipience processes. Educational Psychologist, 52(1), 1737. doi.org/10.1080/00461520.2016.1207538

Montoya, D. M., Hincapié, N. C. \& Carmona, N. D. M. (2016). Enfrentando la ausencia de los padres: recursos psicosociales y construcción de bienestar. Revista Colombiana de Ciencias Sociales, 7(1), 181-200. doi.org/10.21501/22161201.1274 
